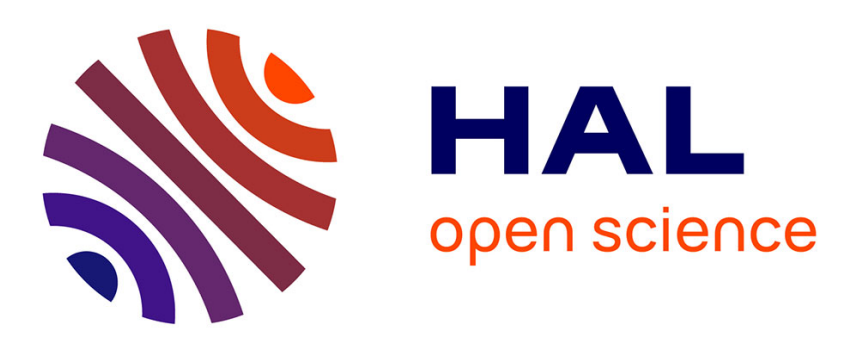

\title{
Large-eddy-simulation-based analysis of Reynolds-stress budgets for a round impinging jet
}

Arthur Colombié, Emmanuel Laroche, François Chedevergne, Remi Manceau, Florent Duchaine, Laurent Gicquel

\section{- To cite this version:}

Arthur Colombié, Emmanuel Laroche, François Chedevergne, Remi Manceau, Florent Duchaine, et al.. Large-eddy-simulation-based analysis of Reynolds-stress budgets for a round impinging jet. Physics of Fluids, 2021, 33 (11), pp.115109. 10.1063/5.0064009 hal-03423068

\section{HAL Id: hal-03423068}

\section{https://hal-univ-pau.archives-ouvertes.fr/hal-03423068}

Submitted on 9 Nov 2021

HAL is a multi-disciplinary open access archive for the deposit and dissemination of scientific research documents, whether they are published or not. The documents may come from teaching and research institutions in France or abroad, or from public or private research centers.
L'archive ouverte pluridisciplinaire HAL, est destinée au dépôt et à la diffusion de documents scientifiques de niveau recherche, publiés ou non, émanant des établissements d'enseignement et de recherche français ou étrangers, des laboratoires publics ou privés. 


\section{Large-Eddy-Simulation-based analysis of Reynolds-stress budgets for a round impinging jet \\ A. Colombié,, a) E. Laroche, ${ }^{1, b)}$ F. Chedevergne, ${ }^{1, c)}$ R. Manceau, ${ }^{2, d)}{ }^{1,}$. Duchaine, ${ }^{3, e)}$ and L. Gicquel ${ }^{3, f)}$ \\ 1) ONERA/DMPE, Université de Toulouse, F-31055 Toulouse, France \\ ${ }^{2)}$ CNRS, Université de Pau et des Pays de l'Adour, E2S UPPA, INRIA, équipe CAGIRE, LMAP, 64000 Pau, \\ France \\ ${ }^{3)}$ CERFACS, 42 Avenue Gaspard Coriolis, 31057 Toulouse, France \\ (Dated: 8 October 2021)}

A large eddy simulation is used to assess the Reynolds-stress budgets of a round impinging jet, in the context of secondmoment closure of turbulence. The present work focuses on the stagnation region where no data are available in the literature except in the wall vicinity. Inside the stagnation region, it is shown that the pressure terms are dominant in the budgets. They balance the Reynolds-stress production and the convective fluxes. A visualization of this equilibrium through a specific indicator reveals that impingement effects extend to less than one nozzle diameter in the wall normal direction and to about one diameter radially. This study underlines the role of the pressure diffusion term that conveys energy to the wall, balancing the high production rates (both positive or negative). Finally, the failure of turbulence models is explained by the absence of appropriate modeling of this pressure diffusion term leading to excessive Reynolds-stress values inside the impingement region.

Keywords: Large eddy simulation, Turbulence modeling, Reynolds-stress budgets, Pressure diffusion

\section{INTRODUCTION}

Because of their high heat transfer efficiency, turbulent impinging jets are commonly used in a large variety of applications, such as de-icing piccolo tubes, blade cooling systems or material tempering methods. Understanding the mechanisms at play is of prime interest and is still an open question. Additionally, this configuration remains a challenging test case for turbulence models, since it embraces many flow features despite a simple geometry, and causes strong discrepancies between standard turbulence closure predictions and available data. Reynolds-stress transport models have been shown to be promising candidates but still suffer from a lack of validation regarding this flow configuration. Second-moment budgets are required to understand the discrepancies between the physical processes and their modeling. Hence, the purpose of the present paper is to provide such reliable statistics by means of a large eddy simulation (LES), for the canonical axisymmetric configuration of Baughn \& Shimizu ${ }^{1}$. This new computation is based on the work of Aillaud et al. ${ }^{2}$.

The complexity of impinging jets resides in the different coexisting flow regions as illustrated in Fig. 1. Far from the plate, the behavior is similar to a free developing jet. The mixing layer grows due to the strong shear between the main stream and the quiescent environment. Above the plate, the fluid expands radially in the wall jet region. There, the mean velocity is parallel to the wall and decreases gradually with the radius $r$. Similarly to the free jet mixing layer, this region

a)Electronic mail: arthur.colombie@onera.fr ${ }^{b)}$ Corresponding author: emmanuel.laroche@onera.fr

c) Electronic mail: francois.chedevergne @onera.fr

${ }^{d)}$ Electronic mail: remi.manceau@univ-pau.fr

e) Electronic mail: florent.duchaine@ cerfacs.fr

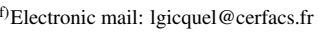

exhibits high shear strain values. Nevertheless, the literature mostly focuses on the impingement area, which is characterized by strong streamline curvature and high normal strain values. Another feature is the high heat transfer rates that occur at the wall. Moreover, the flow in this area displays negative turbulence production close to the wall, due to the positive radial velocity gradient (Nishino et al. ${ }^{3}$, Geers et al. ${ }^{4}$, Shekhar $\&$ Nishino $^{5}$ ).

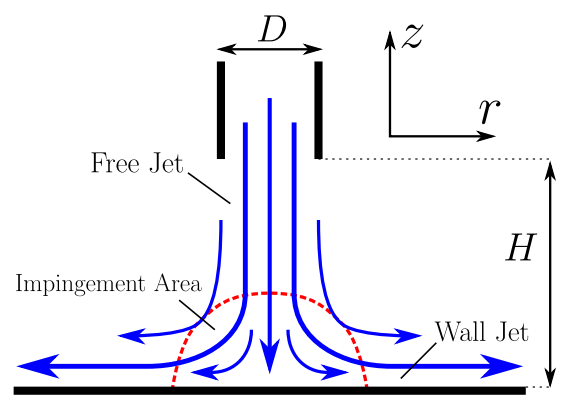

FIG. 1: Different flow regions of an impinging jet

The experiment of Baughn \& Shimizu ${ }^{1}$ has set the pace for heat transfer studies of round impinging jets. For small impingement distances $(H / D<4)$, it revealed the existence of a secondary peak in the Nusselt number distribution, confirmed later by Lee $\&$ Lee $^{6}$ or Brevet et al. ${ }^{7}$, among others. This particular behavior is the cornerstone of numerous studies trying to understand the underlying physics. Afterwards, the pioneering work of Cooper et $_{\text {al. }}{ }^{8}$ provided the mean flow properties (velocity and Reynolds stresses) corresponding to the same configuration, by means of hot wire measurements. These statistics were updated by Geers et al. ${ }^{4}$ using Particle Image Velocimetry (PIV) and Laser Doppler Anemom- 
etry (LDA). More recently, Tummers et al. ${ }^{9}$ also conducted LDA measurements inside a $H / D=2$ impinging jet. Féno et al. ${ }^{10}$ replicated Baughn \& Shimizu's configuration as a validation case for their infrared thermography apparatus and a complete experimental study (dynamics and heat transfer) was conducted by Kim ${ }^{11}$. Grenson et al. ${ }^{12}$ completed the picture, documenting velocity and Reynolds-stress tensor fields for a heated, $H / D=3$, impinging jet.

Many numerical studies attempted to capture and explain the underlying mechanisms of the secondary peak of the Nusselt number. Among these studies, the LES of Hadžiabdic $\&$ Hanjalic $^{13}$ and the direct numerical simulation (DNS) of Dairay et al. ${ }^{14}$ highlighted the effects of vortical structures, causing local unsteady separation/reattachment and enhancing heat transfer. On the other hand, Uddin et al. ${ }^{15}$ and Aillaud et al. ${ }^{2}$ showed the appearance of "hot spots" using LES. These spots are linked to the unsteady thickening of the thermal boundary layer due to elongated structures, causing upwashing/downwashing of the heated/cooled fluid. Both phenomena are recovered in the study of Grenson \& Deniau ${ }^{16}$ but the authors stated that the 'streak-like' downwash process is the most common.

The dynamics of the vortical structures and their interactions with the plate are also observed experimentally. Grenson et al. ${ }^{12}$ and Yadav \& Agrawal ${ }^{17}$ observed that the primary vortex emerging from the shear layer induces an acceleration of the near-wall fluid, associated with cold fluid entrainment from the ambient. A deceleration of the fluid ahead of the primary structure that leads to separation is also noticed. Nevertheless, no secondary structure was visible in Grenson et al. nor in the higher Reynolds number experiment of Yadav \& Agrawal. Both authors concluded to the need of an increase in the spatial resolution of the PIV measurements to capture the formation of the secondary vortex. Yadav \& Agrawal also observed that these structures appear only for small nozzleto-plate spacing since the primary structure looses its intensity when the jet is issued far from the plate, diminishing the probability of separation.

Although insightful, time resolved simulations and experiments remain restricted to academic configurations. Industrial applications involve complex geometries and wall-bounded flows. They therefore require the use of turbulence models, in the Reynolds-averaged Navier-Stokes (RANS) numerical simulation framework, to reduce the computational cost. Nevertheless, several studies (Ashforth-Frost \& Jambunathan ${ }^{18}$ Behnia et al. ${ }^{19}$, Craft et al. ${ }^{20}$, Zuckerman \& Lior $^{21}$ ) revealed that standard two-equation models fail to predict the heat transfer near the stagnation point. The overestimation is attributed to an over-prediction of the turbulent kinetic energy (TKE) at the impingement. Kato \& Launder ${ }^{22}$ as well as Menter ${ }^{23}$ mainly pointed out the defect of Boussinesq's relation that poorly reproduces the turbulence production in this area, i.e. the stagnation point anomaly. Indeed, the modeled production term writes $P_{k}=2 v_{t} S_{i j} S_{i j}$ (with $v_{t}$ the turbulent eddy viscosity and $S_{i j}$ the strain-rate tensor), which is not consistent with the negative value for this term at the impingement and with its exact expression which is linear in $S_{i j}$. Durbin ${ }^{24}$ also highlighted the unexpected large value of the turbulent time scale which diminishes the dissipation rate production, causing the increase of turbulence.

Hence, a variety of corrections for two-equation models were derived. Some of them aim to linearize the production term by a limitation on the time scale $\left(\right.$ Durbin $\left.^{24}\right)$ or by the modification of the eddy-viscosity constant to include strainrate effects (Guimet \& Laurence ${ }^{25}$ ). On the other hand, Kato \& Launder ${ }^{22}$ as well as Menter ${ }^{23}$ suggested the replacement of the strain rate by the vorticity tensor $\left(W_{i j}\right)$ in the TKE production, to diminish its influence for irrotational flows: $W_{i j} \approx 0$ such that $P_{k} \approx 0$ at the impingement. Yap ${ }^{26}$ and Behnia et al. ${ }^{19}$ added length scale corrections to fix the overestimation of $v_{t}$ at the impingement. Craft $e t$ al. ${ }^{27}$ got rid of Boussinesq's relation limitations with a cubic eddy-viscosity model. This resulted in low turbulence values at the impingement and greatly enhanced the heat transfer prediction. Behnia et al. ${ }^{19}$ highlighted the efficiency of Durbin' ${ }^{28} \overline{v^{2}}-f$ model in predicting impinging flows. Actually, the turbulent time and length scale bounds associated with the damping of the wall normal velocity limit turbulence production. Nevertheless, most of these ad hoc corrections mainly obviate the core issue of correctly representing the anisotropy within the impingement area.

Therefore, the use of second-moment closures appears to be a solution to the stagnation point anomaly because these models directly transport the Reynolds-stress components. Indeed, the production term is not modeled. Nonetheless, Craft et al. ${ }^{20}$ showed that basic models exhibit a behavior similar to two-equation models, with large values of TKE and an overestimation of heat transfer. Furthermore, the use of an elliptic blending function, equivalent to the $\overline{v^{2}}-f$ formulation, surprisingly maintains erroneous values (Manceau et al. ${ }^{29}$ ). Nevertheless, Craft $e t a l . .^{20}$ also proved that the use of a wall reflection mode ${ }^{30}$ could significantly improve the predictions. This correction was however designed to fit the results without taking into account the underlying physics of the impinging jet, which questions the behavior of such a remedy for other stagnation flows.

The main curb to the improvement of second-moment closures for round impinging jets lies in the lack of knowledge concerning the Reynolds-stress budgets in the impingement area. In the pioneering work of Nishino et al. ${ }^{3}$, the configuration consists in a confined jet originating six diameters $(H / D=6)$ above the impingement plate at a moderate Reynolds number $(R e=13,000)$. The authors provided the TKE budgets in the mixing layer, very close to the wall and at the edge of the impingement area. A balance between the negative TKE production and the pressure diffusion process was observed in the immediate vicinity of the impingement surface. Indeed, the term $D_{k}^{p}-\varepsilon$, obtained as the residual of the budget was positive. Numerical simulations (LES) by Uddin ${ }^{31}$ and Hadžiabdic ${ }^{32}$ also confirmed the importance of pressure diffusion in the vicinity of the wall, for $H / D=2$ impinging jets. On the other hand, the experiments of Alekseenko et al. ${ }^{33,34}$ provided the TKE budgets at the upstream limit of the impingement area for $H / D=3$ jets. The recent study of Shekhar \& Nishino ${ }^{5}$ investigated the budgets in the stagnation area for a confined $H / D=6$ round impinging jet, at small Reynolds number $(R e=5,200)$. 
The authors provided budgets along horizontal lines located at $z / D=\{0.065 D ; 0.26 D ; 1.0 D\}$ from the impingement plate. Once again, the pressure diffusion process seemed to balance the negative TKE production. The authors also pointed out that the pressure diffusion behavior was not consistent with Lumley's hypothesis ${ }^{35}$, which assumes a proportionality with turbulent diffusion. They concluded on the need for numerical approaches to study pressure-strain and pressure diffusion effects. A large eddy simulation of isotropic turbulence impinging on a leading edge was conducted by Xiong \& $\mathrm{Lele}^{36}$ The budgets for the diagonal Reynolds stresses were provided along the stagnation line. A negative production term was obtained for the transverse component of the Reynolds-stress tensor and the authors pointed out the dominant role of the pressure term that redistributes energy from the streamwise to the other diagonal Reynolds stresses. The dissipation is found to be a minor contributor to the budgets of both streamwise and transverse Reynolds stresses.

The present study aims at providing the Reynolds-stress budgets inside the stagnation region of a turbulent round impinging jet. Those budgets are required to ease secondmoment closure developments for such flows. Indeed, there is only a few investigations on the subject and the measurements available inside the stagnation region focus on small Reynolds numbers and on medium impingement distances. Moreover, the experimental budgets are incomplete since the pressure terms are not measured and cannot be dissociated from dissipation. On the other hand, existing numerical simulation budgets are restricted to the wall vicinity and the effects of the impingement are not studied far from the wall. For this reason, a LES is conducted to get the Reynolds-stress budgets inside the whole impingement region. The canonical configuration of Baughn and Shimizu $(R e=23,000$ and $H / D=2)$ is chosen since it is a reference test case for turbulence model validation, and there is no existing experiment covering secondmoment budgets for this configuration. Therefore, providing these statistics is of interest for Reynolds-stress-modeling.

In the following, a first part is dedicated to the description of the case and the simulation. The mean flow fields are also discussed. In a second part, the Reynolds-stress budget is introduced together with the post-processing methods for the computation of the different terms. Then, a section is devoted to the validation of the budgets and a focus is made on the pressure terms near the stagnation point. Finally, the budgets are analyzed in the impingement area and a discussion arises on the modeling of pressure diffusion.

\section{LES OF A ROUND IMPINGING JET}

The experiment of Baughn \& Shimizu ${ }^{1}$ (with $H / D=2$ ) is often cited as a reference test case for turbulence model assessment. It consists in an impinging jet emerging from a round nozzle (see Fig. 1). The jet is issued from a long tube, providing a fully developed flow at the outlet, located 2 diameters above the plate $(H / D=2)$. The Reynolds number of the flow, based on the inflow bulk velocity and the nozzle diameter, is about 23,000 . The impingement surface is heated with a uniform heat flux and the Nusselt number distribution is determined using liquid crystal temperature measurements.

In order to improve turbulence modeling in the stagnation region of this configuration, the Reynolds-stress budgets are necessary. Thus, a wall-resolved $\left(y^{+}<3.5\right)$ large eddy simulation is performed. It is based on the work of Aillaud et al. ${ }^{2}$ which gives results in good agreement with the experimental data of Tummers et al..$^{9}$ Their finer mesh is kept as well as the boundary conditions in order to obtain the same results for the velocity and Reynolds-stress fields. However, this new simulation focuses on the Reynolds-stress budgets, not computed in Aillaud et al.

The calculation is performed in a $3 D$ axi-symmetric domain (Fig. 2), meshed with 42 million tetrahedral/prism elements. It extends 3 diameters in the radial direction and the nozzleto-plate distance is equal to $2 D$, in accordance with Baughn \& Shimizu's reference case. The inflow pipe is also meshed 2 diameters upstream the nozzle.

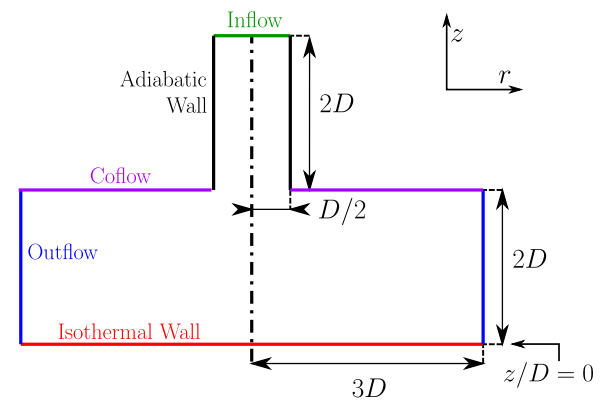

FIG. 2: Representation of the computational domain \& boundary conditions

The inflow boundary condition is set up with a power law velocity profile,

$$
\frac{U(r)}{U(r=0)}=\left(1-\frac{2 r}{D}\right)^{1 / 7.23},
$$

according to the work of Lodato et al. ${ }^{37}$ The turbulent behavior is obtained by supplementing the mean flow with isotropic fluctuations based on Kraichnan's generation method ${ }^{38}$, in order to retrieve the Passot-Pouquet spectrum:

$$
E(k)=16 \sqrt{\frac{2}{\pi}} \frac{\bar{u}^{2}}{k_{0}}\left(\frac{k}{k_{0}}\right)^{4} e^{-2\left(k / k_{0}\right)^{2}},
$$

where $k$ is the wavenumber, $\overline{u^{\prime}}$ the root-mean-square of the fluctuations and $k_{0}$ the peak location in the spectrum. The average bulk velocity is chosen to retrieve the Reynolds number corresponding to Baughn \& Shimizu reference case $(R e=$ $23,000)$, with a low-Mach number $(M a \sim 0.1)$. The nozzle boundary condition is an adiabatic no-slip wall. In order to prevent calculation divergence, a coflow boundary condition is applied on the upper border of the computational domain. It consists of a mean velocity equal to $5 \%$ of the inflow speed, 
without fluctuations. The atmospheric pressure is prescribed on the outflow boundary condition. The plate is an isothermal $\left(T_{\text {wall }}=T_{j e t}\right)$ no-slip wall in order to focus on the dynamics and avoid any thermal bias. Non-reflective boundary conditions (NSCBC) are used for inflow/coflow and outflow (Poinsot \& Lele ${ }^{39}$, Granet et al. ${ }^{40}$ ).

The simulation is performed with the unstructured, parallel code AVBP, developed by CERFACS. It is designed for compressible LES computations. It is based on a cell-vertex finitevolume formulation. The convective terms are computed with a third-order accurate finite element Taylor-Galerkin method $\left(\mathrm{TTGC}^{41}\right)$ and a second-order Galerkin approach is used for the diffusive fluxes. The subgrid-scale model used in the computation for the momentum equation is the Wall Adapting Local Eddy-viscosity (WALE ${ }^{42}$ ) model. The subgrid-scale turbulent heat flux is modeled by a Simple Gradient Diffusion Hypothesis (SGDH) with a constant turbulent Prandtl number. Nevertheless, as the plate is maintained at the jet temperature, thermal effects are small and the flow dynamics is not influenced by temperature variations.

\section{REMINDERS AND MEAN FLOW FIELDS}

The computation of the Reynolds-stress budgets is done through an extra-run of Aillaud $\mathrm{et} \mathrm{al.}{ }^{2}$ large-eddy simulation. Except the $30 \mathrm{~K}$ difference prescribed between the wall and the fluid in Aillaud et al. work, the two simulations are identical. In order to give an insight on the upstream mean flow, a visualization of the mean properties is available on Fig. 3 at $z / D=1.0$.

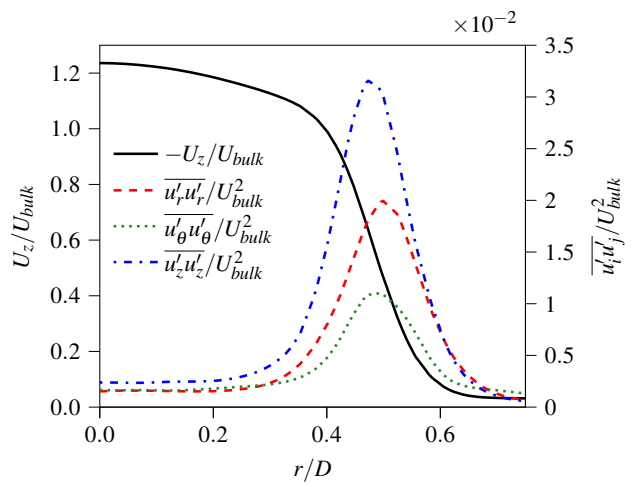

FIG. 3: Profile of the mean flow in the jet core at the location $z / D=1.0$ (present results)

A grid convergence study was conducted by Aillaud $e t$ al. using two meshes based on tetrahedral and prism elements of 21 and 42 millions cells respectively. The wall resolution of the coarse mesh is given by $y^{+} \in[2 ; 5]$ and $r^{+} \in[8 ; 40]$ and the size of the fine mesh element is in the range $y^{+} \in[1 ; 3.5]$ and $r^{+} \in[5 ; 20]$. It was shown that the results are equivalent in the whole jet except for $r / D=1.0$, for the wall-normal Reynoldsstress component. The influence of the subgrid-scale model was also studied with no visible effect.

The main characteristics of the flow are recovered in the present simulation as shown on Fig. 4 for the mean velocity.

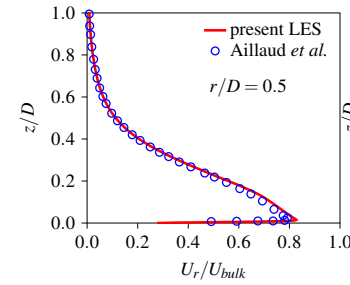

(a)

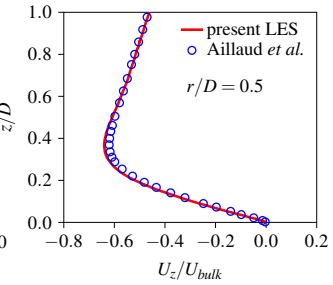

FIG. 4: Comparison of the present results with Aillaud et al. ${ }^{2}$ data for $r / D=0.5$; (a) radial velocity; (b) axial velocity

The Reynolds stresses inside the stagnation area of the jet are shown, along the centerline, on Fig. 5. The observed turbulence intensities are larger near the stagnation point than their asymptotic values, inside the cylindrical duct. Moreover, the agreement between the simulation and the experimental data $^{8,9}$ is good. In particular, the near wall damping of $\overline{u_{z}^{\prime} u_{z}^{\prime}}$ and the sharp rise of $\overline{u_{r}^{\prime} u_{r}^{\prime}}$ are well predicted. These findings confirm the turbulent behavior of the stagnation area in the present simulation.

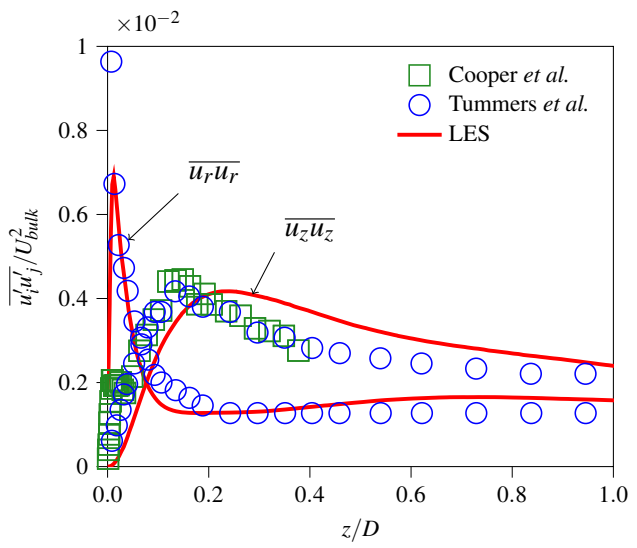

FIG. 5: Comparison of the present results with measurements ${ }^{8,9}$ of the Reynolds stresses along the centerline 


\section{BUDGETS \& POST-PROCESSING}

\section{A. Reynolds-stress budgets}

Using the standard Reynolds-averaged decomposition, the instantaneous field $(u)$ is split into its averaged value $(U)$ and its fluctuating part $\left(u^{\prime}\right): u=U+u^{\prime}$. The exact Reynolds-stress transport equation then reads

$$
\frac{\partial \overline{u_{i}^{\prime} u_{j}^{\prime}}}{\partial t}+C_{i j}=P_{i j}+\phi_{i j}+D_{i j}^{t}+D_{i j}^{p}+D_{i j}^{v}-\varepsilon_{i j} .
$$

The terms in Eq. (3) are defined as follows, with $v$ the kinematic viscosity of the fluid:

$$
\begin{aligned}
& C_{i j}=U_{l} \frac{\partial \overline{u_{i}^{\prime} u_{j}^{\prime}}}{\partial x_{l}}: \text { Convection, } \\
& P_{i j}=-\overline{u_{i}^{\prime} u_{l}^{\prime}} \frac{\partial U_{j}}{\partial x_{l}}-\overline{u_{j}^{\prime} u_{l}^{\prime}} \frac{\partial U_{i}}{\partial x_{l}}: \text { Production, } \\
& \phi_{i j}=\overline{\frac{p^{\prime}}{\rho}\left(\frac{\partial u_{i}^{\prime}}{\partial x_{j}}+\frac{\partial u_{j}^{\prime}}{\partial x_{i}}\right)}: \text { Redistribution, } \\
& D_{i j}^{t}=-\frac{\partial}{\partial x_{l}}\left[\overline{u_{i}^{\prime} u_{j}^{\prime} u_{l}^{\prime}}\right]: \text { Turbulent diffusion, } \\
& D_{i j}^{p}=-\frac{\partial}{\partial x_{l}}\left[\overline{\frac{p^{\prime}}{\rho}\left(u_{i}^{\prime} \delta_{j l}+u_{j}^{\prime} \delta_{i l}\right)}\right] \text { : Pressure diffusion, } \\
& D_{i j}^{v}=\frac{\partial}{\partial x_{l}}\left[v \frac{\partial \overline{u_{i}^{\prime} u_{j}^{\prime}}}{\partial x_{l}}\right]: \text { Viscous diffusion, } \\
& \varepsilon_{i j}=2 v \overline{\frac{\partial u_{i}^{\prime}}{\partial x_{l}} \frac{\partial u_{j}^{\prime}}{\partial x_{l}}}: \text { Dissipation. }
\end{aligned}
$$

Undoubtedly, the budgets obtained from the LES are subject to the filtering effects of the subgrid-scale model. However, since LES resolves a large part of the energetic scales, the terms computed from the resolved variables can be considered as reasonable approximation of the terms that would be given by a direct numerical simulation, except for the dissipation which is linked to the small scales. The reliability of the computed terms based on LES will be assessed agains available experimental data in Sec. V. The dissipation term is not computed directly but estimated from the sum of the other terms.

Moreover, since viscous diffusion is negligible away from the wall and balances $\varepsilon_{i j}$ in the vicinity of the wall, it is convenient to cluster it with dissipation in order to ease the budget analysis. Then, the estimate for the viscous terms is

$$
D_{i j}^{v}-\varepsilon_{i j}=C_{i j}-P_{i j}-\phi_{i j}-D_{i j}^{t}-D_{i j}^{p}
$$

\section{B. Post-processing}

All the terms in the Reynolds-stress transport equation consist in correlations of time-dependent variables [c.f. Eq. (4)]
Their calculation is carried out using the decomposition

$$
\begin{aligned}
& \overline{a^{\prime} b^{\prime}}=\overline{a b}-\bar{a} \bar{b} \\
& \overline{a^{\prime} b^{\prime} c^{\prime}}=\overline{a b c}+2 \bar{a} \bar{b} \bar{c}-\overline{a b} \bar{c}-\overline{a c} \bar{b}-\bar{a} \overline{b c} .
\end{aligned}
$$

The averaged values are obtained using long-time averaging, based on 280,000 time steps $\left(d t=3.85 \times 10^{-7} \mathrm{~s}\right)$, which represents 17.68 convective time scales $\left(\tau_{\text {conv }}=H / U_{\text {bulk }}\right)$. To achieve statistical convergence, the variables are also averaged in the azimuthal direction using 500 samples of the full $3 D$ domain. This requires that all tensor fields are previously projected onto the cylindrical coordinates.

Since the fluxes involve a divergence operator, they need an a posteriori gradient calculation and summation. This may introduce undesirable post-processing artefacts. Thus, the pressure-diffusion term is computed using the classic decomposition

$$
\begin{aligned}
& \underbrace{\left.\frac{\partial}{\partial x_{l}\left[-\overline{p^{\prime}}\left(u_{i}^{\prime} \delta_{j l}+u_{j}^{\prime} \delta_{i l}\right)\right.}\right]}_{D_{i j}^{p}}=\underbrace{-\frac{1}{\rho}\left(\overline{u_{i}^{\prime} \frac{\partial p^{\prime}}{\partial x_{j}}}+\overline{u_{j}^{\prime} \frac{\partial p^{\prime}}{\partial x_{i}}}\right)}_{\Pi_{i j}} \\
& -\underbrace{\overline{p^{\prime}\left(\frac{\partial u_{i}^{\prime}}{\partial x_{j}}+\frac{\partial u_{j}^{\prime}}{\partial x_{i}}\right)}}_{\phi_{i j}}
\end{aligned}
$$

because $\Pi_{i j}$ and $\phi_{i j}$ do not require post-processing divergence calculation. It is worth mentioning that the term $\Pi_{i j}$ also represents the total contribution of the pressure effects to the Reynolds-stress budget, which is in itself an interesting quantity.

Any tensor $T_{i j}$ of the budgets is also converted in dimensionless form using Eq. (8), i.e., based on the inlet bulk velocity $U_{b u l k}$ and the nozzle diameter $D$. Note that in the following, the notation $T_{i j}$ is used for the dimensionless values, for the sake of simplicity.

$$
T_{i j}^{(\text {dim. less })}=T_{i j} D / U_{b u l k}^{3}
$$

\section{COMPARISONS WITH EXISTING DATA}

\section{A. Comparisons with experimental data}

To ensure the reliability of the present study, it is necessary to assess the ability of the simulation to provide a correct estimate of the dominant terms of the Reynolds-stress transport equation. To this aim, the budgets are compared to available experimental data.

Particle Image Velocimetry measurements were conducted by Alekseenko et al. ${ }^{33,34}$. They collected the TKE budget in the free jet area of a $H / D=3$ impinging jet, one diameter above the impingement. Two Reynolds number are considered $(R e=7,600$ and $R e=8,900)$. Based on their velocity and RMS measurements, they computed the production, convection and turbulent diffusion terms. The dissipation was 
indirectly estimated and the pressure diffusion is obtained as the remaining term of the budget.

As seen in Fig. 6, a significant variation of the convection term is encountered in the measurements, on the inner side of the mixing layer. There is also a significant spreading of the data for dissipation and pressure diffusion ( $c f$. Fig. 7). Indeed, there may be a greater uncertainty on the estimated dissipation, linked with the assumptions for its calculation. The pressure diffusion reflects the variability of both the convection and dissipation since it is computed as the remaining term of the budget.

The present LES budgets are extracted at the location $z / D=0.45$ (i.e. $1.55 D$ from the nozzle) and are compared with the data of Alekseenko et al. ( $2.0 \mathrm{D}$ from the nozzle). The locations are different in order to get the same mixing layer width between the computation and the measurements. Indeed, the present Reynolds number is about three times larger than in the experiments of Alekseenko et al. and the nozzle velocity profiles are different. Consequently, the mixing layer growth rates are not the same.

The LES results are in good agreement with the measured terms ( $c f$. Fig. 6). Moreover, the convection term fits with the higher Reynolds-number experiment, in accordance with the higher Reynolds-number of the present LES. Actually, since the Reynolds numbers of the experiments are relatively close, the behavior of $C_{k}$ for $R e=7,600$ is somewhat surprising in the inner side of the mixing layer. The consistency between the simulation and the estimated terms from the measurements $\left(D_{k}^{p}, \varepsilon\right)$ is less straightforward (cf. Fig. 7). This might be linked to the uncertainty on the measured dissipation and the variability of the deduced pressure diffusion. However, the amplitudes appear to be reasonably captured by the LES for both terms.

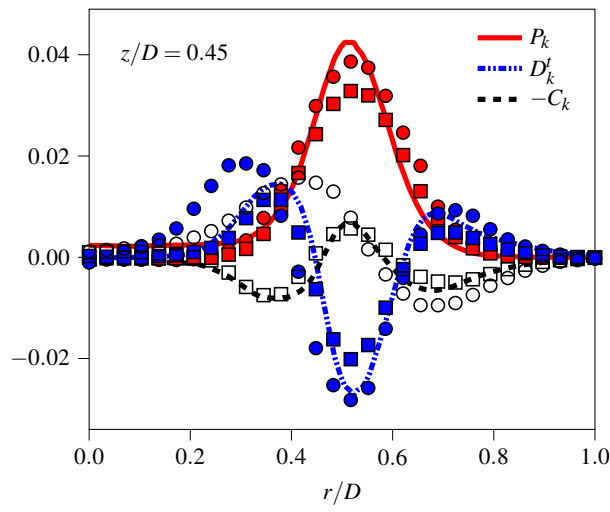

FIG. 6: Comparison with measurements of the TKE budget in the mixing layer of the free jet area Curves: Present study $(z / D=0.45)$ Symbols: data from Alekseenko et al. ${ }^{33,34}$ $(\bigcirc: R e=7,600 ; \square: R e=8,900)$

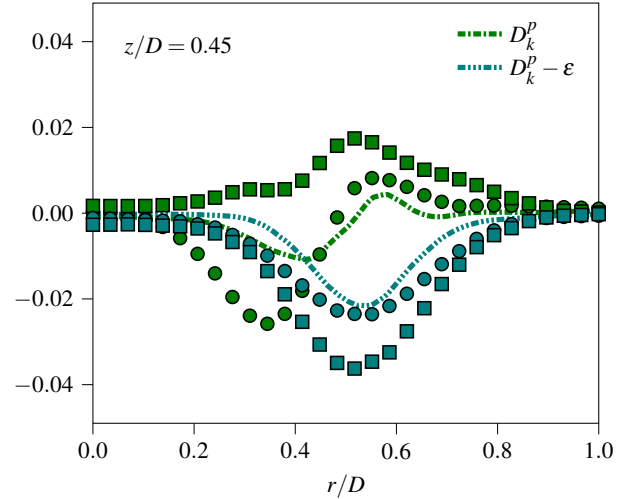

FIG. 7: Comparison with the estimated terms of the TKE budget in the mixing layer of the free jet area Curves: Present study $(z / D=0.45)$ Symbols: data from Alekseenko et al. ${ }^{33,34}$ $(\bigcirc: \operatorname{Re}=7,600 ; \square: \operatorname{Re}=8,900)$

By means of Laser Doppler Anemometry, Toutiaei et al. ${ }^{43}$ measured the individual Reynolds-stress budgets in the mixing layer of a free jet. Their measurements were taken in a plane located 2.5 diameters after the nozzle, in the mixing layer of the potential core region. The production, the convection and the turbulent diffusion terms are directly measured. The other terms (i.e. redistribution, pressure diffusion and viscous terms) are obtained as the residual of the budget.

The present LES budget is extracted at the location $z / D=$ 0.5 (i.e. $1.5 D$ from the nozzle) and is compared with the data of Toutiaei et al. (2.5D from the nozzle). Similarly to the experiments of Alekseenko et al., the mixing layer growth rate are different and no width match is obtained between the computation and the measurements of Toutiaei et al. The Reynolds number is also much larger $(R e=94,000)$. Then, in order to compare the results, the amplitudes of the budgets are scaled using the maximum of their production term : $T_{i j}^{*}=T_{i j} / \max \left(P_{i j}\right)$ with $T_{i j}$ any contributor to the budget. The radial location of the mixing layer is also corrected, in order to get the production maxima at $r^{*} / D=0.5$.

In Fig. 8, the focus is on the budget of the streamwise Reynolds-stress component: $\overline{u_{z}^{\prime} u_{z}^{\prime}}$. The agreement between the LES and the measured terms is satisfactory. Consequently, the overall residual is also well predicted. Under the assumption that both viscous term and pressure diffusion amplitudes are reasonably captured ( $c f$. comparison with Alekseenko et al. data in Fig. 7), it can be expected that the redistribution amplitude is correctly predicted.

The present analysis shows that the LES budgets are in satisfactory agreement with the free jet measurements. Therefore, it confirms that the filtering effect of the subgrid-scale model can be neglected in the prediction of the dominant terms of the budgets. 


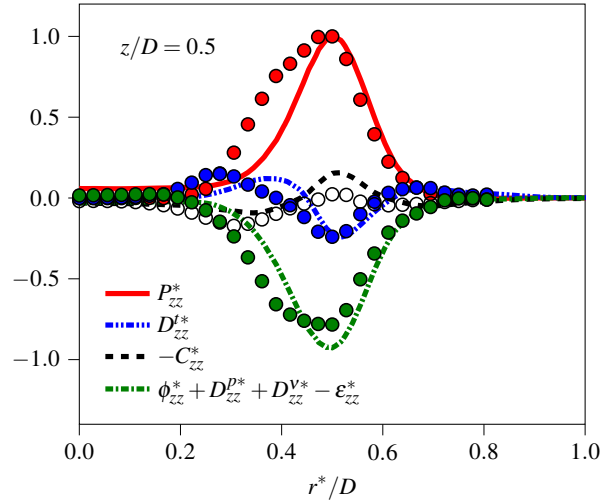

FIG. 8: Comparison with $\overline{u_{z}^{\prime} u_{z}^{\prime}}$ budget measurements in the mixing layer of the free jet area

Curves: Present study $(z / D=0.5)$

Symbols: data from Toutiaei et al. ${ }^{43}$

\section{B. Near-wall behavior analysis}

Hadžiabdic ${ }^{32}$ conducted a LES of the same configuration as the present paper (Baughn \& Shimizu test case, $H / D=2$, $R e=23,000$ ). The near-wall budgets are provided (i.e. for $z / D<\{0.06 ; 0.2\})$. The aim of the present section is to compare the predictions of Hadžiabdić with the present LES budgets in order to validate the computation of the dominan terms. Thus, the focus is on the near-wall budgets in the radia jet $(r / D=2)$ and inside the impingement area $(r / D<0.5)$.

In the radial jet region, the dominant contributors to both $\overline{u_{r}^{\prime} u_{r}^{\prime}}$ and $\overline{u_{z}^{\prime} u_{z}^{\prime}}$ budgets show a correct agreement with the data from Hadžiabdić ( $c f$. Fig. 9). Nonetheless, the two datasets are very dissimilar in the impingement region. There are major discrepancies in the behavior of the evaluated pressure terms, with opposite signs and tendencies near the centerline (cf. Figs. 10 and 11).

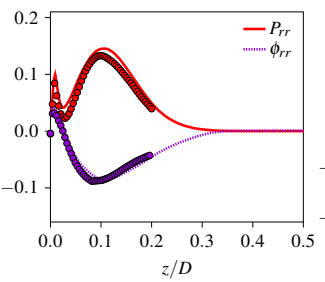

(a)

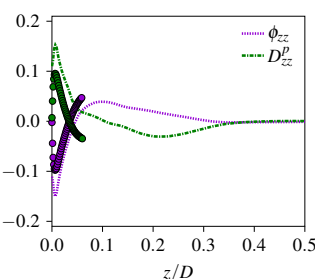

(b)
FIG. 9: Major contributors to $\overline{u_{r}^{\prime} u_{r}^{\prime}}$ budget (a) and $\overline{u_{z}^{\prime} u_{z}^{\prime}}$ budget (b) in the radial jet at $r / D=2.0$

Lines: Present study; Symbols: data from Hadžiabdić 32

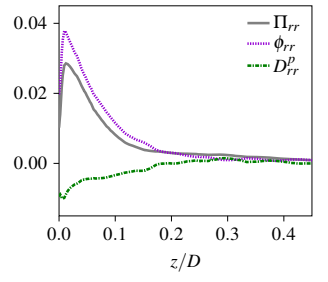

(a)

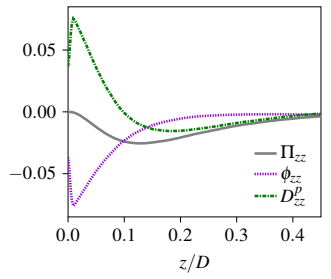

(b)
FIG. 10: Near wall pressure terms along the centerline for $\overline{u_{r}^{\prime} u_{r}^{\prime}}$ (a) and $\overline{u_{z}^{\prime} u_{z}^{\prime}}(\mathrm{b})$ (present simulation)

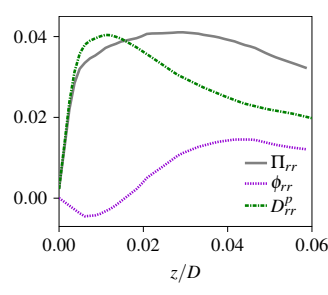

(a)

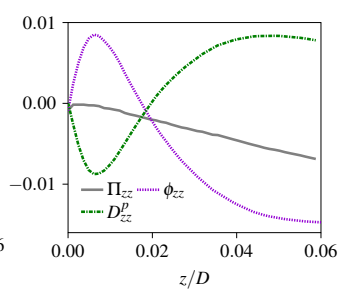

(b)
FIG. 11: Near wall pressure terms along the centerline for $\overline{u_{r}^{\prime} u_{r}^{\prime}}(\mathrm{a})$ and $\overline{u_{z}^{\prime} u_{z}^{\prime}}(\mathrm{b})$ (data from Hadžiabdić ${ }^{32}$ )

To discriminate these two type of results, the near wall behavior of the pressure terms might be recovered by the following phenomenological developments.

\section{Phenomenological analysis}

One may consider instantaneous vortices in the wall vicinity as sketched in Fig. 12. When a vortex approaches the wall, it generates an instantaneous impingement region on one side and a lift up area on the other. The pressure fluctuations shall be positive and negative, respectively. On the impingement side, the velocity fluctuation is negative and goes to zero at the wall. Thus, the wall-normal gradient $\partial u_{z}^{\prime} / \partial z$ is negative. Similarly, on the lifting side, the velocity fluctuation is positive and so is the gradient $\partial u_{z}^{\prime} / \partial z$. Consequently, the correlation $p^{\prime} \partial u_{z}^{\prime} / \partial z$ remains negative whatever the considered region, such that $\phi_{z z}<0$

Near the centerline, the axi-symmetric condition imposes $\overline{u_{r}^{\prime} u_{r}^{\prime}} \equiv \overline{u_{\theta}^{\prime} u_{\theta}^{\prime}} \Rightarrow \phi_{r r} \equiv \phi_{\theta \theta}$. The sign of $\phi_{r r}$ is obtained using the redistributive nature of the pressure-strain term: $2 \phi_{r r}=-\phi_{z z}>0$.

This near wall behavior of the redistributive term reflects the energy transfer caused by the wall blocking effect.

Now, the near wall behavior of $\Pi_{i j}$ is examined from both a phenomenological analysis and theoretical developments. From Fig. 12d, it exists local vertical and radial pressure fluc- 


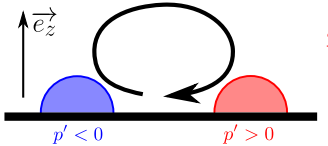

(a)

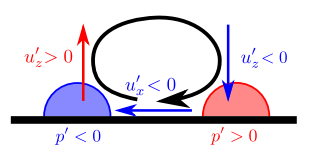

(c)

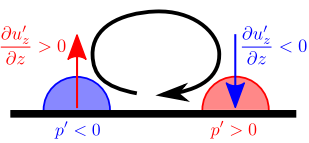

(b)

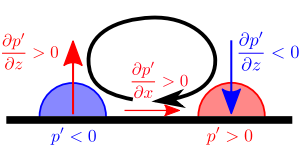

(d)
FIG. 12: Wall blocking effects ; (a) pressure fluctuations ; (b) fluctuating velocity gradients ; (c) velocity fluctuations ; (d) fluctuating pressure gradients

tuations gradients that affect velocity fluctuations (Fig. 12c). The sign of $\Pi_{i j}$ reflects this interaction. It is positive if $u_{i}^{\prime}$ is of the opposite sign of $\partial p^{\prime} / \partial x_{j}$. On the contrary, $\Pi_{i j}$ is negative if $\partial p^{\prime} / \partial x_{j}$ and $u_{i}^{\prime}$ are oriented in the same direction. This reasoning provides an indication on the near wall behavior for both $\Pi_{z z}$ and $\Pi_{r r}$, respectively negative and positive.

In the vicinity of the wall, the sign of $\Pi_{z z}$ can be obtained through Taylor-series expansions of the velocity and pressure fluctuating fields as in the study of Manceau \& Hanjalić ${ }^{44}$ :

$$
\begin{gathered}
u=a_{1} z+a_{2} z^{2}+O\left(z^{3}\right), \\
w=b_{2} z^{2}+O\left(z^{3}\right) .
\end{gathered}
$$

The pressure term writes

$$
\Pi_{z z}=-4 v \overline{b_{2}^{2}} z^{2}+O\left(z^{3}\right) \leq 0 .
$$

From the phenomenological analysis, the following trends [Eq. (11)] are expected. Moreover, the near-wall behavior of $\Pi_{z z}$ is confirmed by the asymptotic development.

$$
\begin{gathered}
\Pi_{r r} \geq 0 \\
\Pi_{z z} \leq 0 \\
\phi_{r r} \geq 0 \\
\phi_{z z} \leq 0
\end{gathered}
$$

The behavior of $\Pi_{i j}$ predicted by the simulation of Hadžiabdić (Fig. 11) is in agreement with the previous developments Nevertheless, the signs of $\phi_{i j}$ components are in contradiction with the phenomenological analysis. On the contrary, the present results (Fig. 10) are consistent with the abovementioned behaviors. Therefore, the near-wall budgets obtained through this new simulation are considered as satisfactory.

\section{RESULTS \& DISCUSSION}

The purpose of this section is to analyze the Reynolds-stress budgets in the impingement area, in order to give an insight into the limitations of turbulence models, which is a prerequisite for their future improvement.

In this context, the focus is first made on the centerlinevicinity $(r / D=0.01)$ budget for $\overline{u_{z}^{\prime} u_{z}^{\prime}}$, as represented in Fig. 13. The influence of the plate appears around $z / D=0.5$. The stagnation point induces an axial deceleration of the flow and a large production (through the term $-\overline{u_{z}^{\prime} u_{z}^{\prime}} \partial U_{z} / \partial z$ ) in the outer part of the impingement zone (i.e. $z / D \in[0.1,0.5]$ ). This source term is mainly balanced by the pressure diffusion process, which transports the energy produced in this region toward the wall. Indeed, $D_{z z}^{p}$ changes sign at $z / D=0.1$ : it is a sink in the outer region and a source in the inner region (i.e. $z / D<0.1$ ). There, the energy income (through pressure diffusion) is mainly transfered to the other diagonal components of the Reynolds-stress tensor by the pressure strain $\left(\phi_{z z}\right)$. The production still contributes to the budget but remains smaller compared to $\phi_{z z}$ and $D_{z z}^{p}$. The convection is a minor contributor to the budget inside the whole impingement area whereas the other terms $\left(D_{z z}^{t}, D_{z z}^{v}\right.$ and $\left.\varepsilon_{z z}\right)$ are negligible.

The positive contribution of pressure diffusion, in the vicinity of the wall, was pointed out in some studies such as Nishino et al. ${ }^{3}$ and Shekhar \& Nishino ${ }^{5}$ for confined round jets.

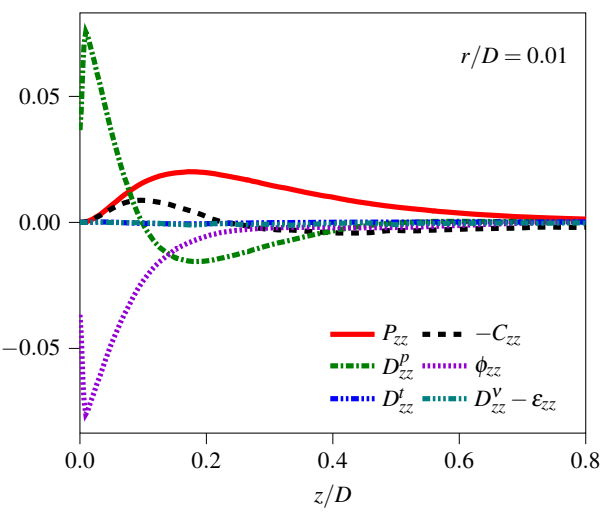

FIG. 13: Centerline $\overline{u_{z}^{\prime} u_{z}^{\prime}}$-budget in the impinging area

The near-wall energy redistribution is recovered in the centerline evaluation of the radial Reynolds-stress budget (Fig. 14). One may note that the amplitude is divided by 2 due to the axi-symmetric condition $\left(\phi_{r r} \equiv \phi_{\theta \theta}\right)$. This peak is balanced by the negative contribution of the production term. The convection and the pressure diffusion also extract energy from the centerline region. The other contributors to the budgets are again negligible.

The energy extracted near the centerline from the radial Reynolds stress is recovered in the region around the impinge- 


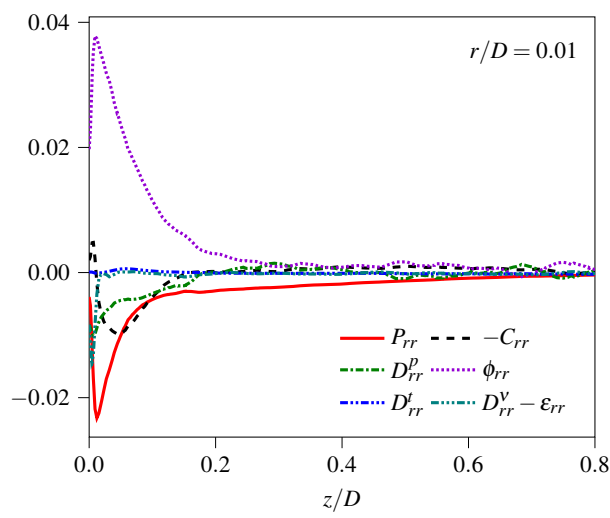

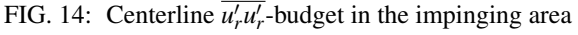

ment area. Fig. 15 presents the budget taken at $r / D=0.3$ for this component of the Reynolds-stress tensor. The balance is similar to the centerline one, apart from the pressure diffusion which acts as a source term in this region. Once again, the changing sign of the pressure diffusion term (between $r / D=0$ and $r / D=0.3$ locations) corresponds to the radial energy transfer by this phenomenon. This had been underlined earlier by Hadžiabdić ${ }^{32}$ for the TKE budget in the near-wall region. However, the positive value of pressure diffusion at this location is also due to the radial diffusion from the surrounding mixing layer of the wall jet. One may notice as well the influence of the mixing layer of the free jet, with an increase of turbulent diffusion far from the wall, above $z / D=0.2$.

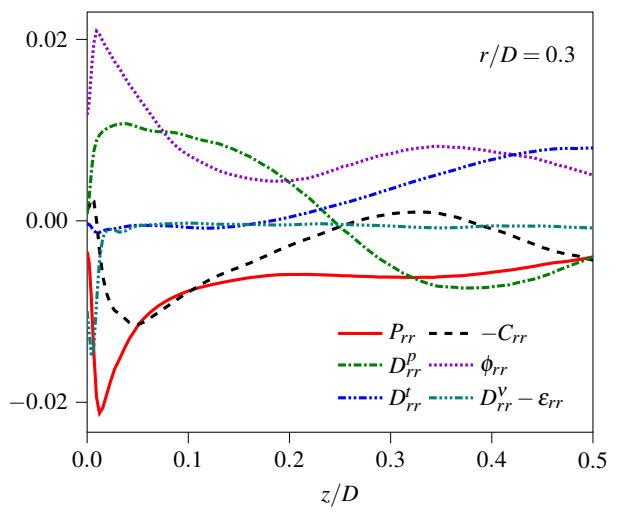

FIG. 15: $\overline{u_{r}^{\prime} u_{r}^{\prime}}$-budget along $r / D=0.3$

From the above examination, the area surrounding the stagnation point (up to $z / D=0.5$ on the centerline) is character- ized by an equilibrium between pressure effects and both the productive and convective terms. Pairing both pressure terms in their original form $\Pi_{i j}=\phi_{i j}+D_{i j}^{p}$ makes it possible to describe a simple behavior for all three diagonal components of the Reynolds-stress tensor as

$$
\Pi_{\alpha \alpha} \approx C_{\alpha \alpha}-P_{\alpha \alpha} .
$$

Obviously, the balance (12) is also obtained for the turbulent kinetic energy as shown in Fig. 16, but the redistributive nature of the pressure strain simplifies the original expression to

$$
\Pi_{k} \equiv D_{k}^{p} \approx C_{k}-P_{k}
$$

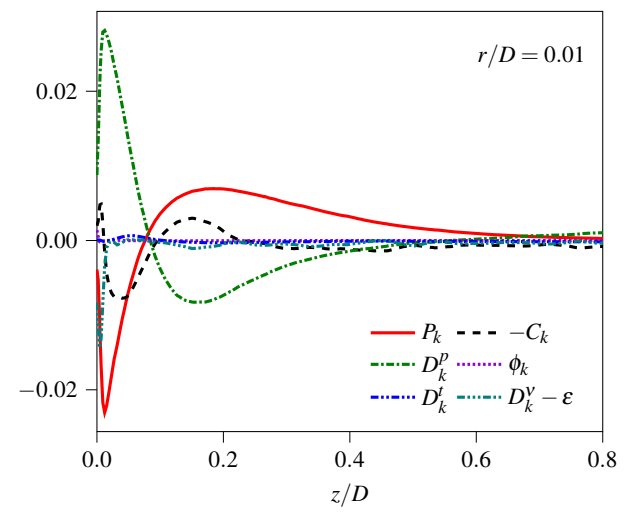

FIG. 16: Centerline TKE budget in the impinging region

Hence, the pressure diffusion is the main mechanism which transfers the turbulent kinetic energy to the impingement area, from the productive (upper) region toward the (lower) destructive $\left(P_{k}<0\right)$ one.

The extent of impingement effects is examined through a visualization of the equilibrium regions. To do so, an equilibrium indicator $\zeta_{i j}$ is constructed. It is expressed as the ratio of both sides of Eq. (12), leading to the form (14). This indicator approaches the value $\zeta_{i j}=1$ when equilibrium is reached.

$$
\zeta_{i j}=\frac{\Pi_{i j}}{C_{i j}-P_{i j}}
$$

Since this indicator is highly sensitive to small budget values, it is applied to the dominant-Reynolds-stress budget $\overline{u_{z}^{\prime} u_{z}^{\prime}}$-budget) and shown in Fig. 17. Nonetheless, similar results are obtained with the other normal components. Areas with low turbulence intensities are shown in gray since the indicator is irrelevant. The equilibrium is observed in an approximately conical region that extends radially up to $r / D=1.0$ and up to $z / D=0.75$ in the wall normal direction. 


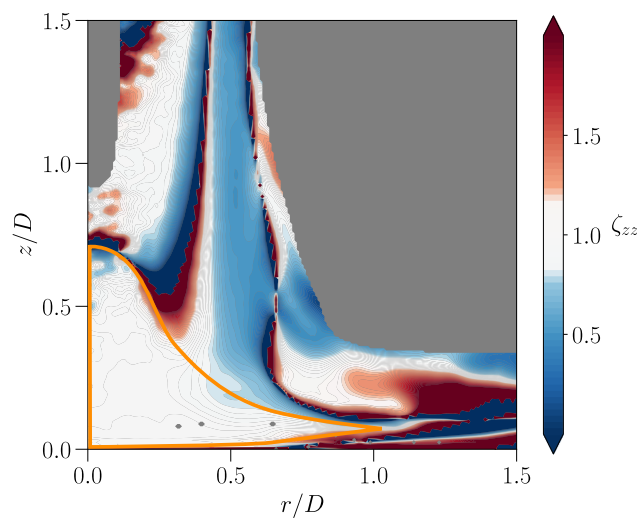

FIG. 17: Equilibrium indicator $\zeta_{z z}$

This region, highlighted by the orange contour, is hereafter considered as the impingement-equilibrium area. Note that the equilibrium is also valid in the jet core although not studied here.

To sum up, the impingement mechanisms may be sketched as in Fig. 18. The wall normal deceleration causes an important production of $\overline{u_{z}^{\prime} u_{z}^{\prime}}$ below $z / D=0.5$, the extra energy being brought down to the wall by pressure diffusion. There, it is redistributed to other components and partly absorbed by the mean flow due to the negative production term. The oversupplied energy is then mainly transported outward by convection.

Therefore, the correct modeling of the pressure diffusion is a key to improve stagnation flow predictions of RANS turbulence models.

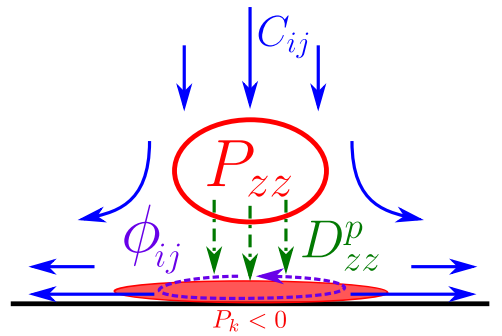

FIG. 18: Scheme of the energy transfers through the impingement area

Lumley's hypothesis is often cited as a way to take pressure diffusion terms into account in turbulence models. In its very first proposal ${ }^{35}$, it reads

$$
\overline{p^{\prime} u_{i}^{\prime}}=\frac{2}{5} \overline{k^{\prime} u_{i}^{\prime}}
$$

Doing so, a proportionality is obtained between the pressure diffusion term and the turbulent one. As a consequence, the pressure term is included within the model for the turbulent diffusion.

The previous impinging region analysis demonstrates that pressure diffusion is one of the major terms in the budgets while turbulent diffusion remains negligible. This behavior is obviously inconsistent with Lumley's hypothesis. The underestimation of the diffusive effects of the pressure term explains the overestimation of the TKE in all standard turbulence closures.

\section{CONCLUSION}

A large eddy simulation has been conducted to explore the Reynolds-stress budgets for impinging flows. Actually, these statistics give an insight in the physical processes inside the stagnation region, which may serve as a basis for secondmoment-closure improvements. The chosen configuration is the canonical $H / D=2$ round impinging jet, popularized by the work of Baughn \& Shimizu ${ }^{1}$

First, the ability of the LES to reproduce the dominant terms of the budgets is assessed. The results in the free jet region are compared with experimental data and the agreement with the measurements is satisfactory. Within the radial jet, the results are compared to another LES of the same configuration. The major terms are also well reproduced. Although LES is not as accurate as direct numerical simulation, these comparisons provide sufficient confidence in the representativity of the budgets to conduct a relevant analysis of the relative importance of the terms and their role in turbulence dynamics. An analysis of the near wall behavior of the pressure terms is also conducted to confirm the reliability of the results. Subsequently, the Reynolds-stress budgets inside the impingement region are analyzed. It is shown that production and convection are balanced by the pressure terms, as described by Eq. (12). An appropriate equilibrium indicator is defined, which highlights that this equilibrium extends in a conical region that goes up to $z / D=0.75$ along the axis and $r / D=1$ near the wall. Among the pressure terms, pressure diffusion is of particular importance since it conveys energy from the outer region to the wall vicinity. This behavior counterweights the turbulence production linked to the wall normal deceleration. Finally, pressure diffusion modeling by Lumley's ${ }^{35}$ hypothesis is discussed. It assumes a proportionality between pressure and turbulent diffusion. However, the present study shows that turbulent diffusion is overwhelmed by pressure diffusion in the stagnation region.

These results explain the inability of RANS models to capture the dynamics of the impingement. Indeed, the pressure diffusion is underestimated near the stagnation point, leading to an overestimation of the turbulent kinetic energy. In order to correctly reproduce the dynamics of the flow, the equilibrium (12) needs to be recovered. In that respect, the modification proposed by Kato \& Launder ${ }^{22}$ or Menter ${ }^{23}$ might be seen as zeroth order corrections. Indeed, the modification of the production term implicitly approximates the equilibrium (i.e. 
it is equivalent to add a term for $\Pi_{k} \equiv D_{k}^{p}=-2 v_{t} S_{i j}\left(S_{i j}-W_{i j}\right)$ which balances $P_{k}$ inside the stagnation region and equals zero otherwise). The use of a more elaborate model, including pressure diffusion effects, may greatly enhance secondmoment-closure efficiency for the reproduction of stagnation flows and, in particular, for the prediction of heat transfer on such configurations.

${ }^{1}$ J. Baughn and S. Shimizu, "Heat transfer measurements from a surface with uniform heat flux and an impinging jet," Journal of Heat Transfer (Transactions of the ASME (American Society of Mechanical Engineers), Series C);(United States) 111 (1989)

${ }^{2}$ P. Aillaud, F. Duchaine, L. Gicquel, and S. Didorally, "Secondary peak in the nusselt number distribution of impinging jet flows: A phenomenological analysis," Physics of Fluids 28, 095110 (2016).

${ }^{3}$ K. Nishino, M. Samada, K. Kasuya, and K. Torii, "Turbulence statistics in the stagnation region of an axisymmetric impinging jet flow," International Journal of Heat and Fluid Flow 17, 193-201 (1996).

${ }^{4}$ L. Geers, M. Tummers, and K. Hanjalić, "Experimental investigation of impinging jet arrays," Experiments in Fluids 36, 946-958 (2004).

${ }^{5} \mathrm{C}$. Shekhar and K. Nishino, "Turbulence energetics in an axisymmetric impinging jet flow," Physics of Fluids 31, 055111 (2019).

${ }^{6} \mathrm{~J}$. Lee and S.-J. Lee, "Stagnation region heat transfer of a turbulent axisymmetric jet impingement," Experimental Heat Transfer 12, 137-156 (1999).

${ }^{7}$ P. Brevet, E. Dorignac, and J. Vullierme, "Mach number effect on jet impingement heat transfer," ANNALS-NEW YORK ACADEMY OF SCIENCES 934, 409-416 (2001).

${ }^{8}$ D. Cooper, D. Jackson, B. Launder, and G. Liao, "Impinging jet studies for turbulence model assessment-i. flow-field experiments," International Journal of Heat and Mass Transfer 36, 2675-2684 (1993).

${ }^{9} \mathrm{M}$. Tummers, J. Jacobse, and S. Voorbrood, "Turbulent flow in the near field of a round impinging jet," International Journal of Heat and Mas Transfer 54, 4939-4948 (2011).

${ }^{10} \mathrm{M}$. Fénot, J.-J. Vullierme, and E. Dorignac, "Local heat transfer due to several configurations of circular air jets impinging on a flat plate with and without semi-confinement," International journal of thermal sciences $\mathbf{4 4}$ 665-675 (2005)

${ }^{11} \mathrm{~N}$. Kim, Analyse expérimentale d'un jet turbulent impactant sur un plaque plane et sur un obstacle de section carrée, Ph.D. thesis, Toulouse 3 (2005).

${ }^{12}$ P. Grenson, O. Léon, P. Reulet, and B. Aupoix, "Investigation of an impinging heated jet for a small nozzle-to-plate distance and high reynolds number: An extensive experimental approach," International Journal of Heat and Mass Transfer 102, 801-815 (2016)

${ }^{13} \mathrm{M}$. Hadžiabdić and K. Hanjalić, "Vortical structures and heat transfer in round impinging jet," Journal of Fluid Mechanics 596, 221-260 (2008)

${ }^{14}$ T. Dairay, V. Fortuné, E. Lamballais, and L. Brizzi, "Direct numerical simulation of a turbulent jet impinging on a heated wall," Journal of Fluid Mechanics 764, 362-394 (2015).

${ }^{15} \mathrm{~N}$. Uddin, S. Neumann, and B. Weigand, "LES simulations of an impinging jet: On the origin of the second peak in the nusselt number distribution," International Journal of Heat and Mass Transfer 57, 356-368 (2013)

${ }^{16} \mathrm{P}$. Grenson and H. Deniau, "Large-eddy simulation of an impinging heated jet for a small nozzle-to-plate distance and high reynolds number," International Journal of Heat and Fluid Flow 68, 348-363 (2017).

${ }^{17} \mathrm{H}$. Yadav and A. Agrawal, "Effect of vortical structures on velocity and turbulent fields in the near region of an impinging turbulent jet," Physics of Fluids 30, 035107 (2018).

${ }^{18} \mathrm{~S}$. Ashforth-Frost and K. Jambunathan, "Numerical prediction of semiconfined jet impingement and comparison with experimental data," International Journal for Numerical methods in fluids 23, 295-306 (1996)

${ }^{19}$ M. Behnia, S. Parneix, and P. Durbin, "Prediction of heat transfer in an axisymmetric turbulent jet impinging on a flat plate," International journa of heat and mass transfer 41, 1845-1855 (1998).

${ }^{20} \mathrm{~T}$. Craft, L. Graham, and B. Launder, "Impinging jet studies for turbulence model assessment-ii an examination of the performance of four turbulence models," International Journal of Heat and Mass Transfer 36, 2685-2697 (1993)

${ }^{21}$ N. Zuckerman and N. Lior, "Jet impingement heat transfer: physics, corre- lations, and numerical modeling," Advances in Heat Transfer 39, 565-631 (2006)

${ }^{22} \mathrm{M}$. Kato and B. Launder, "The modelling of turbulent flow around stationary and vibrating square cylinders," (1993) pp. 10-4.

${ }^{23} \mathrm{~F}$. Menter, "Improved two-equation k- $\omega$ turbulence models for aerodynamic flows," Nasa Sti/recon Technical Report N 93, 22809 (1992).

${ }^{24}$ P. Durbin, "On the k- $\varepsilon$ stagnation point anomaly," Int. J. Heat and Fluid Flow 17, 89-90 (1996)

${ }^{25} \mathrm{~V}$. Guimet and D. Laurence, "A linearised turbulent production in the k- $\varepsilon$ model for engineering applications," in Engineering Turbulence Modelling and Experiments 5 (Elsevier, 2002) pp. 157-166.

${ }^{26} \mathrm{C}$. YAP, Turbulent heat and momentum transfer in recirculating and impinging flows(Ph. D. Thesis), Ph.D. thesis, University of Manchester Institute of Science and Technology (UMIST) (1987).

${ }^{27}$ T. Craft, B. Launder, and K. Suga, "Development and application of a cubic eddy-viscosity model of turbulence," International Journal of Heat and Fluid Flow 17, 108-115 (1996)

${ }^{28}$ P. Durbin, "Near-wall turbulence closure modeling without "damping functions"," Theoretical and computational fluid dynamics 3, 1-13 (1991).

${ }^{29}$ R. Manceau, R. Perrin, M. Hadžiabdić, and S. Benhamadouche, "Investigation of the interaction of a turbulent impinging jet and a heated, rotating disk," Physics of Fluids 26, 035102 (2014)

${ }^{30}$ T. Craft and B. Launder, "New wall-reflection model applied to the turbulent impinging jet," AIAA journal 30, 2970-2972 (1992).

${ }^{31} \mathrm{~N}$. Uddin, Turbulence modeling of complex flows in CFD, Ph.D. thesis, Institute of Aerospace Thermodynamics, Universität Stuttgart (2008).

${ }^{32} \mathrm{M}$. Hadžiabdić, LES, RANS and combined simulation of impinging flows and heat transfer, Ph.D. thesis, University of Sarajevo, Bosnia and Herzegovina (2006).

${ }^{33}$ S. Alekseenko, A. Bilsky, V. Dulin, B. Ilyushin, and D. Markovich, "Nonintrusive determination of turbulent energy balance in free and confined jet flows," in TSFP DIGITAL LIBRARY ONLINE (Begel House Inc., 2005).

${ }^{34}$ S. Alekseenko, A. Bilsky, V. Dulin, and D. Markovich, "Experimental study of an impinging jet with different swirl rates," International Journal of Heat and Fluid Flow 28, 1340-1359 (2007)

35. Lumley, "Computational modeling of turbulent flows," in Advances in applied mechanics, Vol. 18 (Elsevier, 1979) pp. 123-176.

${ }^{36} \mathrm{Z}$. Xiong and S. Lele, "Stagnation-point flow under free-stream turbulence," Journal of Fluid Mechanics 590, 1-33 (2007).

${ }^{37}$ G. Lodato, L. Vervisch, and P. Domingo, "A compressible wall-adapting similarity mixed model for large-eddy simulation of the impinging round jet," Physics of Fluids 21, 035102 (2009)

${ }^{38}$ R. Kraichnan, "Diffusion by a random velocity field"” The Physics of Fluids 13, 22-31 (1970)

${ }^{39}$ T. J. Poinsot and S. Lele, "Boundary conditions for direct simulations of compressible viscous flows," Journal of computational physics 101, 104 129 (1992).

${ }^{40}$ V. Granet, O. Vermorel, T. Léonard, L. Gicquel, and T. Poinsot, "Comparison of nonreflecting outlet boundary conditions for compressible solvers on unstructured grids," AIAA journal 48, 2348-2364 (2010).

${ }^{41} \mathrm{O}$. Colin and M. Rudgyard, "Development of high-order taylor-galerkin schemes for les," Journal of Computational Physics 162, 338-371 (2000). ${ }^{42}$ F. Nicoud and F. Ducros, "Subgrid-scale stress modelling based on the square of the velocity gradient tensor," Flow, Turbulence and Combustion 62, 183-200 (1999).

${ }^{43}$ S. Toutiaei, R. Semaan, and J. Naughton, "Reynolds stress and turbulence kinetic energy balances in swirling jets," in 48th AIAA Aerospace Sciences Meeting Including the New Horizons Forum and Aerospace Exposition (2010) p. 105.

${ }^{44}$ R. Manceau and K. Hanjalić, "Elliptic blending model: A new near-wall reynolds-stress turbulence closure," Physics of Fluids 14, 744-754 (2002). 